\title{
Polycystic ovarian syndrome is linked to increased oxidative stress in Omani women
}

This article was published in the following Dove Press journal:

International Journal of Women's Health

\author{
Maha AH Sulaiman' \\ Yahya M Al-Farsi' \\ Maha MAl-Khaduri ${ }^{2}$ \\ Jumana Saleh ${ }^{3}$ \\ Mostafa I Waly 4,5
}

'Department of Family Medicine and Public Health, College of Medicine and Health Sciences, Sultan Qaboos University, Al-Khoudh, Sultanate of Oman; ${ }^{2}$ Department of Obstetrics and Gynecology, College of Medicine and Health Sciences, Sultan Qaboos University, Al-Khoudh, Sultanate of Oman; ${ }^{3}$ Department of Biochemistry, College of Medicine and Health Sciences, Sultan Qaboos University, Al-Khoudh, Sultanate of Oman; ${ }^{4}$ Department of Food Science and Nutrition, College of Agricultural and Marine Sciences, Sultan Qaboos University, Al-Khoudh, Sultanate of Oman; ${ }^{5}$ Nutrition Department, High Institute of Public Health, Alexandria, Egypt
Correspondence: Yahya M Al-Farsi Department of Family Medicine and Public Health, College of Medicine and Health Sciences, Sultan Qaboos University, PO Box 35, PC, 123 Al-Khoudh, Sultanate of Oman

Tel +96824143430

Fax +968 $244 \mid 3300$

Email ymfarsi@squ.edu.om
Purpose: Literature emerging from Western countries has reported increased levels of serum oxidative stress markers among polycystic ovarian syndrome (PCOS) women. In the Arab region, there is limited research about the association between oxidative stress and PCOS. This study aimed to compare sociodemographic and clinical characteristics, sex hormones, and oxidative stress indices between PCOS women and non-PCOS women and to investigate the correlation between oxidative stress biomarkers and sex hormones.

Methods: This hospital-based case-control study was conducted among reproductive-aged women. The study included 51 women diagnosed with PCOS (as per Rotterdam 2003 criteria) and 45 control women who were not diagnosed with PCOS. Serum samples were collected to measure the mean levels of the following sex hormones: total testosterone, dehydroepiandrosterone sulfate, estradiol and progesterone, as well as to measure biomarkers of oxidative stress including glutathione peroxidase (GPx), glutathione reductase (GR), glutathione (GSH), and total antioxidant capacity (TAC).

Results: PCOS women exhibited clinical characteristics including irregular menses, hirsutism, and acne compared to the control group $(P \leq 0.05)$. Significant differences were observed in the waisthip ratio of PCOS women compared to controls $(P=0.004)$. GPx and GR activity levels appeared to be higher among PCOS women compared to controls; however, no statistically significant differences were observed between the two groups $(P>0.05)$. PCOS women had lower GSH and TAC levels compared to controls with a statistically significant difference observed for GSH levels $(P=0.006)$. Correlation analysis showed a significant negative correlation between estradiol and TAC in the total sample $(r=-0.284, P=0.005)$.

Conclusion: This study provides supportive evidence that oxidative stress might play a role in the pathogenesis of PCOS and, hence, oxidative stress parameters could be suggested as diagnostic markers for early diagnosis of high-risk groups. Also, the study provides supportive evidence that obesity and sex hormones, particularly estradiol, in PCOS may contribute to enhanced oxidative stress.

Keywords: PCOS, oxidative stress, Oman, case-control, anthropometric, glutathione

\section{Introduction}

Polycystic ovarian syndrome (PCOS) is a multifactorial endocrine disorder predominantly affecting between $5 \%-10 \%$ of reproductive-aged women. PCOS women commonly have classical clinical features of oligo- or anovulation, obesity, and hyperandrogenism. ${ }^{1}$ Moreover, PCOS can occur with hyperinsulinemia, glucose intolerance, hyperlipidemia, type 2 diabetes mellitus, hypertension, coronary atherosclerosis, and endometrial cancer. ${ }^{2-5}$

Oxidative stress is thought to be involved in the development of various diseases such as cancer, Alzheimer's disease, autism, atherosclerosis, diabetes, heart 
failure, and infection. ${ }^{6-12}$ Oxidative stress refers to the imbalance between oxidants and antioxidants that results in an abnormal redox state of cells. This leads to the assembly of peroxides and free radicals that can damage different components of the cell, including nucleic acids, proteins, lipids, carbohydrates, and other molecules. ${ }^{13,14}$ The two major forms of radicals are reactive oxygen species (ROS) and reactive nitrogen species (RNS). The ROS include superoxide radical, hydrogen peroxide $\left(\mathrm{H}_{2} \mathrm{O}_{2}\right)$, and hydroxyl radical, ${ }^{15,16}$ and the RNS include nitric oxide and its metabolites. ${ }^{16}$

Antioxidants are a class of molecules that can reduce the destructive effects of free radicals. They are of two types: either enzymatic like superoxide dismutase, catalase, glutathione peroxidase (GPx), and glutathione reductase (GR), or non-enzymatic such as glutathione (GSH), $\alpha$-tocopherol (vitamin $\mathrm{E}$ ), ascorbate (vitamin $\mathrm{C}$ ), and $\beta$-carotene. ${ }^{6}$ These antioxidants have been reported to have an important role in the female reproductive system and in the pathogenesis of female infertility. ${ }^{13,17}$

Oxidative stress has been associated with PCOS. ${ }^{18-21} \mathrm{~A}$ meta-analysis study by Murri et al concluded that circulating markers of oxidative stress are abnormal in women with PCOS, suggesting that oxidative stress may participate in the pathophysiology of PCOS. ${ }^{22}$

However, the role of oxidative stress in the pathogenesis of PCOS is not yet fully understood. Studies have suggested that oxidative stress seems to be involved in PCOS by causing altered steroidogenesis in the ovaries, which subsequently contributes to increasing androgen levels, disturbing follicular development, and infertility. ${ }^{23,24}$ Moreover, factors such as obesity, insulin resistance, and cardiovascular risks have shown to be linked to oxidative stress in PCOS women. ${ }^{21,25}$

Although previous studies have reported an association between oxidative stress and PCOS, there is only limited research about the association between oxidative stress and PCOS in the Arab region. This study was carried out in the Sultanate of Oman, an Arab Middle East country located in southwest Asia.

A few studies have been conducted on PCOS in Oman. A hospital-based prevalence study reported 7.1 PCOS cases per 1,000 women between 25-34 years, and the overall standardized prevalence estimate was 2.8 cases per 1,000 women (95\% CI 0.7, 9.6). ${ }^{26}$ Al-Fazari et al found that suboptimal nutritional and dietary practices are common among Omani women with PCOS compared to controls. ${ }^{27}$ Furthermore, we earlier reported higher rates of psychological burden indices among Omani PCOS women compared to controls. ${ }^{28}$ Despite this, the presence of oxidative stress among PCOS women in
Oman has not been explored. Therefore, this study aimed to 1) compare sociodemographic, clinical, and anthropometric characteristics, sex hormones, and oxidative stress indices among women diagnosed with PCOS vs non-PCOS women; and 2) investigate the correlation between oxidative stress biomarkers and sex hormones.

\section{Materials and Methods}

This hospital-based case-control study was conducted from February 2015 to January 2016 at Sultan Qaboos University Hospital (SQUH), a tertiary teaching hospital in Oman. Written informed consent was obtained from the participants and from parents on behalf of the participants who were younger than 18 years. The study was approved by the Medical Research Ethics Committee at College of Medicine \& Health Sciences, Sultan Qaboos University (MREC \# 826).

\section{Participants}

The sample size was calculated by allowing for a level of significance (type 1 error) of 5\% and 95\% CI and with an a priori assumption based on the most recent meta-analysis ${ }^{22}$ that the GSH levels among PCOS cases would be $15 \%$ lower than that of the controls $(0.17$ vs $0.20 \mu \mathrm{mol} / \mathrm{mL})$, with a common standard deviation of 0.05 and a case-to-control ratio of $1: 1$. The power calculation indicated a sample size of 88 participants was required to achieve a power of $80 \%$ for the study. ${ }^{29}$

The study involved 96 women within the age category of 16-49 years: 51 PCOS cases and 45 controls who shared similarities in ethnicity, culture, and quality of care. Convenience sampling and simple random sampling were used to select cases and controls, respectively.

\section{Ascertainment and selection of cases}

The ascertainment of PCOS diagnosis was based on Rotterdam criteria, which defines PCOS syndrome by any two of the following three characteristics: oligo- and/or anovulation, clinical and/or biochemical signs of hyperandrogenism, and polycystic ovaries on ultrasonography with the exclusion of related disorders. ${ }^{30}$ Pregnant, menopausal, or post menopausal women or those with specific tumors or endocrine-related diseases were excluded from this study. A total of 53 eligible participants were identified as PCOS cases from the Obstetrics \& Gynecology (OBGYN) outpatient clinic. The number of PCOS women who agreed to participate was 51, resulting in a response rate of $96 \%$. Two senior gynecologists examined all PCOS cases, and the agreement between the two gynecologists about PCOS ascertainment was $96 \%$. Upon independent review and discussion of 
patients' profiles by the research team, the percentage agreement reached $100 \%$.

\section{Ascertainment and selection of controls}

The enrolled controls were selected from a random sample of 180 potential controls who had visited the OBGYN outpatient clinic during the recruitment phase of the study. Eligible controls included non-pregnant women of reproductive age who were not diagnosed with PCOS and did not have specific tumors or any other endocrine-related diseases.

Based on the random sampling scheme, 59 potential controls were selected. The eligible controls who fulfilled the inclusion and exclusion criteria were 55. Of this group, only 45 were recruited because some refused to participate while others provided incomplete information, yielding a response rate of $82 \%$. Two senior gynecologists clinically examined all controls to exclude the possibility that they may have had subclinical PCOS features. Percentage agreement by both the gynecologists was found to be $96 \%$. The percentage of agreement reached $100 \%$ following an independent review and discussion of the patients' profiles with the research team.

\section{Study tools}

\section{The study questionnaire}

A standardized and validated questionnaire was filled during a face-to-face interview. ${ }^{27}$ The questionnaire included information about: 1) sociodemographics, 2) comorbidities, 3) clinical features, 4) vitamin and mineral intake, and 5) anthropometric measurements.

\section{Anthropometric measurements}

Body weight (in $\mathrm{kg}$ ) and height (in $\mathrm{cm}$ ) measurements were performed using standard procedures. Body mass index (BMI) was calculated as follows: weight $(\mathrm{kg}) /$ height $^{2}\left(\mathrm{~m}^{2}\right)$. BMI reference values were defined as normal, overweight, and obese at $18.5-24.9,25.0-29.9$, and $\geq 30.0 \mathrm{~kg} / \mathrm{m}^{2}$, respectively. ${ }^{31}$

Waist-hip ratio (WHR) and waist circumference (WC) were measured according to standard protocols. As per the World Health Organization, WHR standards for female were considered to indicate low, moderate, and high risk for chronic diseases/metabolic complications at $<0.80$, $0.80-0.84$, and $\geq 0.85$, respectively; and abdominal obesity was defined as a WHR $>0.85$ for females or a BMI $>30.0 \mathrm{~kg} / \mathrm{m}^{2}{ }^{31}$ WC cutoff points for female were reported to infer low, moderate, and high risk for chronic diseases/metabolic complications at $\leq 80.0,80.1-88.0$, and $>88.0 \mathrm{~cm}$, respectively. ${ }^{31}$

\section{Biochemical assays}

A total of $5 \mathrm{~mL}$ of venous blood was withdrawn into a plain tube by a specialized nurse via venipuncture. Blood samples were collected between days 2 and 4 of the menstrual cycle (i.e. follicular phase) to measure total testosterone, dehydroepiandrosterone sulfate (DHEAS), and estradiol levels, and during the middle luteal phase to measure progesterone levels. Among women who had had amenorrhea within the previous 6 months, blood samples were taken for hormonal analysis at random. Sex hormones in the sera were measured by chemiluminescence immunoassay system using UniCel DxI 600 Access (Beckman Coulter, Inc., Brea, CA, USA).

Oxidative biomarkers in the sera were measured for the two groups according to the manufacturer's instructions (Biovision, Inc., Milpitas Boulevard, CA, USA). GPx was measured using a GPx activity colorimetric assay kit (K762-100), GR was measured using a GR activity colorimetric assay kit (K761200), GSH was measured using a glutathione fluorometric assay kit (K264-100), and total antioxidant capacity (TAC) was measured using a TAC colorimetric assay kit (K274-100).

\section{Data analysis}

Significant statistical differences between the proportions of categorical data were evaluated using chi-square analyses. For a small sample size where the expected frequency was less than five in any of the cells in $2 \times 2$ tables, the non-parametric Fisher's exact test (two-tailed) was used. To evaluate the significant differences between the mean values of two continuous variables, the unpaired Student's $t$-test was used. The non-parametric test (Mann-Whitney $U$ test) was used to ascertain significant differences when the assumption of normality was violated. Continuous variables were represented as mean and standard error of the mean (SEM). Pearson's correlation coefficient (r) was calculated to assess correlations between the continuous variables. Wherever appropriate, the non-parametric Spearman's correlation coefficient replaced the Pearson's correlation coefficient. Univariate logistic regression modeling was used to obtain ORs and 95\% CIs, which were taken as measures of association between PCOS and selected risk factors. Multilinear regression modeling was used to obtain standardized $\beta$ coefficients as measures of association between the continuous outcome variables and potential predictors. For all statistical tests, $P \leq 0.05$ was considered statistically significant. All statistical analyses were performed using the Statistical Package for Social Sciences (SPSS) software (version 24.0; IBM Corporation, Armonk, NY, USA), and GraphPad Prism software (version 5.0; GraphPad Software, Inc., San Diego, CA, USA). 


\section{Results}

Overall, a total of 96 Omani women were included in the study, of which there were 51 (53.1\%) PCOS cases and $45(46.9 \%)$ controls. Table 1 shows the sociodemographic characteristics of PCOS cases and controls. The majority of participants were married $(91.7 \%)$ and 51\% were between 26-34 years. The mean age of the case and control groups was $29.9 \pm 6.2$ and $32.2 \pm 6.3$ years, respectively. In addition, $55.2 \%$ had acquired university education and $53.1 \%$ were employed. There were no statistically significant differences between PCOS cases and controls for all the sociodemographic characteristics. Table 1 also shows selected clinical characteristics of participants. Overall, the proportions of women with irregular menses (82.4\%), hirsutism (84.3\%) and acne (29.4\%) were significantly higher in the case group compared to the control group ( $P \leq 0.05)$. In addition, the proportions of women with a history of vitamin $\mathrm{B}_{12}$ and regular folate intake tended to be lower in the case group than those in the control group; however, no statistically significant differences were observed between the two groups $(P>0.05)$.

Table I Sociodemographic and clinical characteristics of the study groups, Oman, 2017

\begin{tabular}{|c|c|c|c|c|}
\hline \multirow[t]{3}{*}{ Characteristics } & Total & Cases & Controls & \multirow[t]{3}{*}{$P$-value } \\
\hline & $(N=96)$ & $(\mathbf{N}=\mathbf{5} I)$ & $(N=45)$ & \\
\hline & n (\%) & n (\%) & n (\%) & \\
\hline Age (years) & & & & 0.43 \\
\hline$\leq 25$ & $19(19.8)$ & II (2I.6) & $8(17.8)$ & \\
\hline $26-34$ & $49(51.0)$ & $28(54.9)$ & $21(46.7)$ & \\
\hline$\geq 35$ & $28(29.2)$ & $12(23.5)$ & $16(35.5)$ & \\
\hline Marital status & & & & 0.14 \\
\hline Unmarried & $8(8.3)$ & $2(3.9)$ & $6(13.3)$ & \\
\hline Married & $88(91.7)$ & $49(96.1)$ & $39(86.7)$ & \\
\hline Education level & & & & 0.63 \\
\hline School educated & $43(44.8)$ & $24(47.1)$ & $19(42.2)$ & \\
\hline University educated & $53(55.2)$ & $27(52.9)$ & $26(57.8)$ & \\
\hline Employment status & & & & 0.21 \\
\hline Employed & $5 \mathrm{I}(53.1)$ & $24(47.1)$ & $27(60.0)$ & \\
\hline Unemployed & 45 (46.9) & $27(52.9)$ & $18(40.0)$ & \\
\hline Monthly family income & & & & 0.68 \\
\hline \multicolumn{5}{|l|}{$(\mathrm{OMR})$} \\
\hline$<400$ & $15(15.6)$ & $6(11.8)$ & $9(20.0)$ & \\
\hline $400-799$ & $26(27.1)$ & $14(27.5)$ & $12(26.7)$ & \\
\hline $800-1,199$ & $18(18.8)$ & II (2I.5) & $7(15.5)$ & \\
\hline$>1,200$ & 37 (38.5) & $20(39.2)$ & 17 (37.8) & \\
\hline Irregular menses & $55(57.3)$ & $42(82.4)$ & $13(28.9)$ & $<0.001$ \\
\hline Presence of hirsutism & $44(45.8)$ & $43(84.3)$ & I (2.2) & $<0.001$ \\
\hline Presence of acne & $20(20.8)$ & $15(29.4)$ & $5(I I . I)$ & 0.04 \\
\hline History of vitamin $B_{12}$ intake & $12(12.5)$ & $6(11.8)$ & $6(13.3)$ & 0.82 \\
\hline History of folate intake & & & & 0.15 \\
\hline Regular supplementation & $32(33.3)$ & $15(29.4)$ & $17(37.8)$ & \\
\hline Irregular supplementation & $50(52.1)$ & $31(60.8)$ & $19(42.2)$ & \\
\hline
\end{tabular}

Abbreviation: OMR, Omani Rials.
Table 2 shows the anthropometric characteristics of the study groups. The proportion of obese women tended to be higher in the PCOS group compared to the control group (52.9\% vs 40.0\%); however, the difference was statistically non-significant $(P>0.05)$. Moreover, the proportion of women with a high risk for chronic diseases/metabolic complications - as reflected by WHR - was significantly higher in the case group compared to the control group (70.6\% vs $37.8 \%, P=0.004)$. A similar pattern was also observed in that the proportion of women with a high risk for chronic diseases/ metabolic complications - as reflected by WC - appeared to be higher in the case group compared to the control group (56.9\% vs $42.2 \%)$. Nonetheless, the difference was not found to be statistically significant $(P>0.05)$.

Also, the OR generated by logistic regression analysis indicated an increasing risk pattern for the anthropometric characteristics of PCOS women. The results showed that PCOS women were at a higher risk for obesity compared to non-PCOS women ( $\mathrm{OR}=1.77,95 \%$ CI 0.65, 4.82). Moreover, a stronger measure of association was observed for a high risk of chronic diseases, as demonstrated by WHR $(\mathrm{OR}=5.51,95 \% \mathrm{CI} 1.69,17.95)$ and $\mathrm{WC}(\mathrm{OR}=2.11,95 \%$ CI 0.84, 5.29), among PCOS women.

Table 3 lists the mean levels of sex hormones and oxidative stress biomarkers among PCOS cases and controls. Testosterone $(P<0.001)$ and DHEAS $(P=0.001)$ levels were significantly higher among PCOS cases compared to controls. Although the levels of estradiol tended to be higher among PCOS cases compared to controls, the difference was statistically non-significant between the two study groups $(P>0.05)$. On the contrary, progesterone levels were significantly lower among PCOS cases compared to controls $(P<0.001)$. In addition, GPx and GR activity levels tended to be higher among PCOS cases compared to controls, with no statistically significant differences between the two groups $(P>0.05)$. Moreover, GSH levels were significantly lower among PCOS cases compared to controls ( $P=0.006$ ) (Figure 1). Similarly, TAC levels tended to be lower among PCOS cases than controls, although the difference observed between the two groups was not statistically significant $(P>0.05)$ (Figure 2$)$.

Table 4 shows the correlation between oxidative stress biomarkers and sex hormones. Generally, a weak correlation was found between oxidative stress biomarkers and sex hormones. The table shows a significant negative correlation between estradiol and TAC $(r=-0.284, P=0.005)$. On the contrary, a positive correlation was found between progesterone and GSH; nonetheless, the correlation was statistically non-significant $(r=0.221, P>0.05)$. 
Table 2 Anthropometric characteristics of the study groups, Oman, 2017

\begin{tabular}{|c|c|c|c|c|c|}
\hline \multirow[t]{3}{*}{ Characteristics } & \multirow{2}{*}{$\frac{\text { Total }}{(\mathrm{N}=96)}$} & \multirow{2}{*}{$\begin{array}{l}\text { Cases } \\
(\mathrm{N}=5 \mathrm{I})\end{array}$} & \multirow{2}{*}{$\frac{\text { Controls }}{(\mathrm{N}=45)}$} & \multirow[t]{3}{*}{ OR (95\% Cl) } & \multirow[t]{3}{*}{$P$-value } \\
\hline & & & & & \\
\hline & n (\%) & n (\%) & n (\%) & & \\
\hline BMI & & & & & 0.47 \\
\hline Normal & $24(25.0)$ & II (2I.6) & $13(28.9)$ & 1.0 & \\
\hline Overweight & $27(28.1)$ & $13(25.5)$ & $14(3||)$. & $1.10(0.36,3.30)$ & \\
\hline Obese & $45(46.9)$ & $27(52.9)$ & $18(40.0)$ & $1.77(0.65,4.82)$ & \\
\hline WHR & & & & & 0.004 \\
\hline Low risk ${ }^{\mathrm{a}}$ & $18(18.8)$ & $5(9.8)$ & $13(28.9)$ & 1.0 & \\
\hline Moderate risk & $25(26.0)$ & $10(19.6)$ & $15(33.3)$ & $1.73(0.47,6.39)$ & \\
\hline High risk ${ }^{\mathrm{a}}$ & $53(55.2)$ & $36(70.6)$ & $17(37.8)$ & $5.51(1.69,17.95)$ & \\
\hline WC & & & & & 0.28 \\
\hline Low risk ${ }^{\mathrm{a}}$ & $31(32.3)$ & $13(25.5)$ & $18(40.0)$ & 1.0 & \\
\hline Moderate risk ${ }^{\mathrm{a}}$ & $17(17.7)$ & $9(17.6)$ & $8(17.8)$ & $1.56(0.47,5.12)$ & \\
\hline High risk ${ }^{\mathrm{a}}$ & $48(50.0)$ & $29(56.9)$ & $19(42.2)$ & $2.11(0.84,5.29)$ & \\
\hline
\end{tabular}

Note: ${ }^{a}$ Risk for chronic diseases/metabolic complications.

Abbreviations: BMI, body mass index; WHR, waist-hip ratio; WC, waist circumference.

Table 5 shows the multilinear regression modeling of the association between TAC levels and each of the sex hormones and anthropometric parameters. Estradiol was the major significant predictor of oxidative stress, as determined by a reduction in TAC levels, after adjusting for all study parameters $(\beta=-0.380$, $P<0.001)$. Among the anthropometric indicators, the regression model illustrated potential associations between decreasing TAC levels and increasing obesity parameters (i.e. BMI and WHR). Nonetheless, these associations were statistically non-significant $(P>0.05)$. This regression model accounted for $20.3 \%$ of the variability in TAC among Omani women, as indicated by the $\mathrm{R}^{2}$ value of the model. Estradiol alone accounted for an $8.1 \%$ of decrease in TAC $\left(\mathrm{R}^{2}=8.1 \%, P=0.005\right)$.

Table 3 Mean levels of sex hormones and oxidative stress biomarkers among the study groups, Oman, 2017

\begin{tabular}{|c|c|c|c|}
\hline \multirow[t]{3}{*}{ Parameter } & \multirow{2}{*}{$\begin{array}{l}\text { Cases } \\
(\mathrm{N}=5 \mathrm{I})\end{array}$} & \multirow{2}{*}{$\begin{array}{l}\text { Controls } \\
(\mathrm{N}=45)\end{array}$} & \multirow[t]{3}{*}{$P$-value } \\
\hline & & & \\
\hline & Mean \pm SEM & Mean \pm SEM & \\
\hline $\begin{array}{l}\text { Testosterone } \\
(\mathrm{nmol} / \mathrm{L})\end{array}$ & $\mathrm{I} .83 \pm 0.8 \mathrm{I}$ & $1.10 \pm 0.60$ & $<0.001$ \\
\hline $\begin{array}{l}\text { DHEAS } \\
(\mu \mathrm{mol} / \mathrm{L})\end{array}$ & $5.31 \pm 2.37$ & $3.80 \pm 1.90$ & 0.001 \\
\hline $\begin{array}{l}\text { Estradiol } \\
(\mathrm{nmol} / \mathrm{L})\end{array}$ & $0.45 \pm 0.63$ & $0.24 \pm 0.24$ & 0.30 \\
\hline $\begin{array}{l}\text { Progesterone } \\
(\mathrm{nmol} / \mathrm{L})\end{array}$ & $15.38 \pm 16.86$ & $34.75 \pm 19.82$ & $<0.00 \mathrm{I}$ \\
\hline GPx (U/L) & $9,174.58 \pm 1,063.20$ & $7,498.73 \pm 639.96$ & 0.43 \\
\hline $\mathrm{GR}(\mathrm{U} / \mathrm{L})$ & $499.92 \pm 79.84$ & $435.12 \pm 25.29$ & 0.25 \\
\hline $\mathrm{GSH}(\mathrm{mg} / \mathrm{mL})$ & $6.32 \pm 1.59$ & II. $.22 \pm 4.22$ & 0.006 \\
\hline $\mathrm{TAC}(\mathrm{mmol} / \mathrm{L})$ & $3.80 \pm 0.12$ & $4.04 \pm 0.14$ & 0.49 \\
\hline
\end{tabular}

Abbreviations: DHEAS, dehydroepiandrosterone sulfate; GPx, glutathione peroxidase; GR, glutathione reductase; GSH, glutathione; TAC, total antioxidant capacity; SEM, standard error of mean.

\section{Discussion}

The present study compared sociodemographic, clinical, and anthropometric characteristics, sex hormones, and oxidative stress biomarkers among PCOS cases and controls. It also investigated correlations between oxidative stress biomarkers and sex hormones.

Our study showed that most of the participants were young, married, and had a university level of education. Both case and control groups were comparable in terms of sociodemographic characteristics, and the observed differences were not statistically significant. This finding signaled that the sociodemographic characteristics were less likely to confound the obtained results. Overall, our study showed that among PCOS women, the distribution of clinical indicators of irregular menses ( $82.4 \%)$, hirsutism (84.3\%), and acne (29.4\%) suggests that

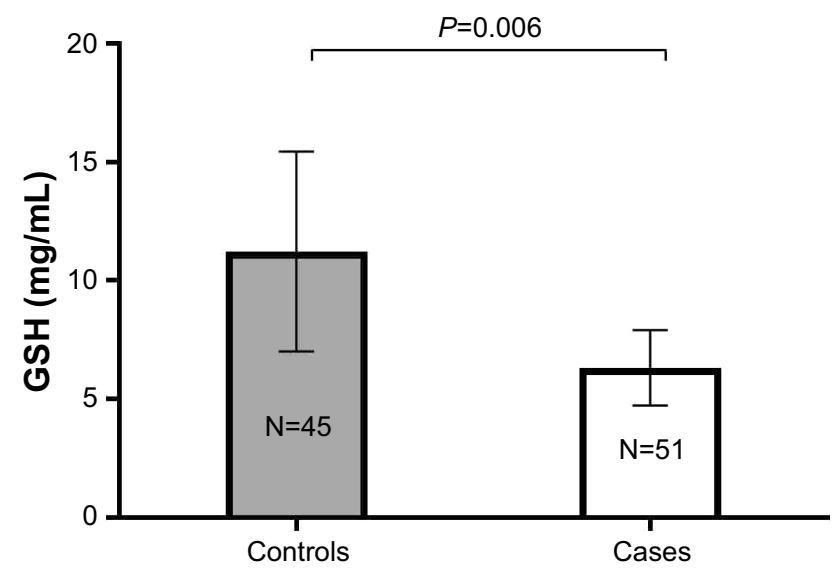

Figure I GSH levels among controls vs PCOS cases.

Note: Results are expressed as mean \pm SEM.

Abbreviations: GSH, glutathione; PCOS, polycystic ovarian syndrome; SEM, standard error of mean. 


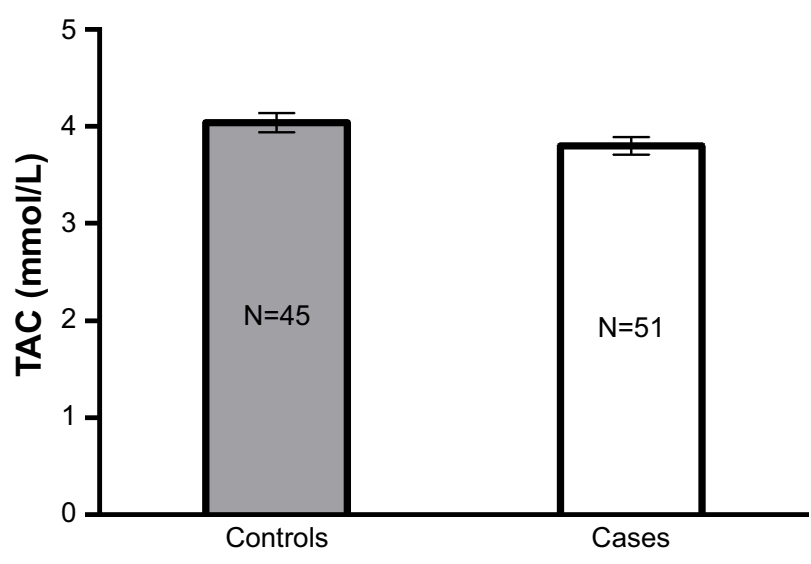

Figure 2 TAC levels among controls vs PCOS cases. Notes: Results are expressed as mean \pm SEM. $P>0.05$.

Abbreviations: TAC, total antioxidant capacity; PCOS, polycystic ovarian syndrome; SEM, standard error of mean.

Omani women with PCOS have a similar phenotype to that of South Asian women with PCOS. As reported by Wijeyaratne et al and Mani et al, South Asian PCOS women had more prevalent acne, hirsutism, and irregular menses compared to Caucasian women with PCOS. ${ }^{32,33}$ This might be explained by the geographical and cultural proximity of Oman to South Asia, resulting in shared genetic and sociocultural factors that may have led people of Oman to demonstrate phenotypic similarities to South Asians.

Our data also showed differential anthropometric characteristics - represented by BMI, WHR, and WC - among PCOS women compared to controls. Our findings are consistent with similar findings from other studies. ${ }^{34,35}$ Also, Al-Fazari et al reported abdominal obesity to be a common feature among PCOS women in Oman. ${ }^{27}$

Our data revealed significantly higher androgen levels (i.e. testosterone and DHEAS) and significantly lower progesterone levels among PCOS women in comparison to nonPCOS women. Our findings are in line with those of previous studies. $^{34,36,37}$ The high androgen levels can be explained by the pathophysiological interactions of the syndrome. Alterations in gonadotropin-releasing hormone $(\mathrm{GnRH})$ or

Table 4 Correlation between oxidative stress biomarkers and sex hormones, Oman, 2017

\begin{tabular}{lllll}
\hline & GPx & GR & TAC & GSH \\
\hline Testosterone & 0.062 & -0.074 & 0.079 & -0.173 \\
DHEAS & 0.080 & -0.061 & 0.045 & -0.170 \\
Estradiol & $-0.15 \mathrm{I}$ & -0.163 & $-0.284^{\mathrm{a}}$ & 0.029 \\
Progesterone & -0.063 & 0.018 & -0.099 & 0.221 \\
\hline
\end{tabular}

Notes: a Correlation is significant. $P=0.005$.

Abbreviations: DHEAS, dehydroepiandrosterone sulfate; GPx, glutathione peroxidase; GR, glutathione reductase; TAC, total antioxidant capacity; GSH, glutathione.
Table 5 Multilinear regression modeling of the association between TAC levels and each of the sex hormones and anthropometric parameters, Oman, 2017

\begin{tabular}{llll}
\hline Predictor & \multicolumn{2}{l}{ Regression statistics } & P-value \\
\cline { 2 - 3 } & Standardized $\boldsymbol{\beta}$ & $\mathbf{S E}$ & \\
\hline Testosterone & 0.049 & 0.132 & 0.68 \\
DHEAS & 0.084 & 0.045 & 0.46 \\
Estradiol & -0.380 & 0.182 & $<0.001$ \\
Progesterone & 0.031 & 0.005 & 0.76 \\
BMI & -0.341 & 0.033 & 0.23 \\
WC & 2.692 & 0.121 & 0.22 \\
WHR & -0.900 & 12.306 & 0.28 \\
\hline
\end{tabular}

Notes: $R^{2}$ value for the full model is $20.3 \% ; R^{2}$ value for the stepwise model (estradiol only) is $8.1 \%$. $(P=0.005)$.

Abbreviations: DHEAS, dehydroepiandrosterone sulfate; BMI, body mass index; WC, waist circumference; WHR, waist-hip ratio; SE, standard error.

insulin secretion may provide a possible explanation for the high androgen levels among those women. ${ }^{38}$ Because PCOS women tend to experience oligo-or anovulation, their progesterone levels tend to be low. Decreased levels of progesterone can also increase the pulse frequency of $\mathrm{GnRH}$, resulting in increased levels of luteinizing hormone, and overproduction of androgens. ${ }^{5}$

GPx is an antioxidant enzyme that reduces $\mathrm{H}_{2} \mathrm{O}_{2}$ and lipid peroxides to water and lipid alcohols and oxidizes GSH to glutathione disulfide (GSSG). ${ }^{39}$ In this study, an increased trend in the activity levels of GPx was found in the case group compared to the control group. Our results tend to be in agreement with those of another study, which reported an increase of GPx levels in tissue samples of PCOS compared to non-PCOS samples. ${ }^{40}$ The apparent decline in GPx activity among controls is likely due to the fact that GPx has higher affinity toward peroxides and thus would scavenge most radicals produced. ${ }^{41}$

GR is an antioxidant enzyme that catalyzes the NADPHdriven reduction of GSSG to GSH. ${ }^{42}$ Slightly higher activity levels of GR were observed among PCOS women. This noticeable trend among PCOS women might have resulted from their greater need for the reduction of oxidized GSH formed by GPx.

GSH is a powerful and abundant antioxidant that plays a key role in protecting cells from oxidative damage. ${ }^{43,44}$ Previous research has reported decreased blood GSH levels in chronic diseases including cancer, and gastrointestinal and cardiovascular diseases. ${ }^{45}$ In the present study, a significant decrease in GSH was observed among women with PCOS compared to non-PCOS women. Our results are in line with those of previous studies, which showed a significant decrease in GSH among PCOS women. ${ }^{21,46}$ GSH reacts with 
ROS by its thiol groups in the reduced state, and therefore prevents the damage of cells. Increased androgen production results in more ROS formation among women with PCOS, which may lead to the depletion of $\mathrm{GSH}^{46}$ The decreased levels of GSH in PCOS cases would possibly require the activation of the redox GSH cycle by activating GPx and GR enzymes to enhance the production of functional GSH.

TAC is an antioxidant biomarker measuring the antioxidant potential of body fluids and has been reported to be significantly low in several diseases. ${ }^{47,48}$ The present data showed a reduced trend in TAC levels among PCOS women compared to the control group. This slight decrease in serum TAC levels might be a reflection of the levels of GSH, which is the main antioxidant in the body. These data tend to be in agreement with those of a previous study in Saudi Arabia, which showed lower TAC levels among PCOS women compared to non-PCOS women. ${ }^{49}$ Hilali et al similarly reported decreased TAC levels among PCOS women compared to the control group. ${ }^{18}$

Although frequent research has been conducted on oxidative stress and hormonal variations among females, no definite conclusion has been reached regarding the association between oxidative stress biomarkers and sex hormones. Some studies have shown positive correlations while others demonstrated negative correlations or no correlations at all. ${ }^{50-56}$ Previously reported findings have indicated that estradiol has an antioxidant effect by enhancing antioxidant enzyme activities or exhibiting free radical scavenging properties. ${ }^{50,57}$ Other findings have linked estradiol with adverse metabolic effects including increased oxidative stress. ${ }^{58-60}$ In our analysis, a significant negative correlation was found between TAC and estradiol. Furthermore, estradiol was the major predictor of oxidative stress among all measured parameters. This suggests that estradiol may have an opposing role to oxidative defense mechanisms. On the other hand, progesterone, which is usually associated with favorable metabolic conditions and known to have antioxidant properties, ${ }^{61,62}$ showed a positive trend with regards to the correlation with $\mathrm{GSH}$, which is a major antioxidant factor.

In this study, increased BMI and WHR among PCOS women appeared to be concomitant with increased estradiol levels and decreased TAC levels. Therefore, obesity maybe linked to increased oxidative stress in women, as a result of female hormone disturbance in PCOS. The prevalence of obesity among females in Oman increased from $23.8 \%$ in the year 2000 to $26 \%$ in 2008 , with $54 \%$ of females being centrally obese. ${ }^{63,64}$ Furthermore, there has been a remarkable change in dietary and lifestyle habits in Oman in recent years, with an increase in the consumption of fast food and sugar-dense beverages and low physical activity levels, which has been accompanied by a remarkable increase in body anthropometric measurements such as BMI and WHR. ${ }^{65}$ In addition, studies suggest that increased mitochondrial oxidative stress can occur as a result of overnutrition and decreased energy demands. Therefore, decreased cellular respiration leads to the accumulation of ROS that remain in the mitochondria, ultimately leading to $\mathrm{H}_{2} \mathrm{O}_{2}$ leakage causing cellular damage. ${ }^{66}$

Evidence from the literature suggests that changes in oxidative status might play a role in the etiology of PCOS. ${ }^{67,68}$ However whether oxidative stress is the cause or an outcome of PCOS requires further exploration.

\section{Limitations}

First, information gathered from the interview could have been affected by recall bias possibly resulting in a misclassification error. Thus, this limitation was overcome by ensuring that any misclassification error which occurred would most probably have been non-differential across the study groups. Second, this study was conducted merely on serum samples and not tissues or cells in which enzyme activities are higher. Therefore, the data obtained from our study are preliminary findings that may provide a useful biochemical basis for further studies on homogenized tissues from target organs. It is worth mentioning that future studies in which tissue samples are obtained from case and control groups should be designed with caution as they might be subject to ethical constraints.

\section{Conclusion}

The study provides supportive evidence that oxidative stress might play a role in the pathogenesis of PCOS, and hence, these parameters may be suggested as diagnostic markers for early diagnosis and screening of high-risk groups. Also, the study provides supportive evidence that obesity and sex hormones, particularly estradiol, in PCOS may contribute to enhanced oxidative stress. Furthermore, rigorous populationbased case-control studies with larger sample sizes and sampling intervals, and the integration of additional variables are needed to further explore the link between PCOS and oxidative stress. Also, conducting laboratory animal studies would contribute to detailed exploration of oxidative stressinduced molecular mechanisms in PCOS.

\section{Acknowledgments}

The authors would like to acknowledge the physicians and nursing staff of OBGYN clinic at SQUH for their valuable support during this study. 


\section{Disclosure}

The authors report no conflicts of interest in this work.

\section{References}

1. Azziz R, Woods KS, Reyna R, Key TJ, Knochenhauer ES, Yildiz BO. The prevalence and features of the polycystic ovary syndrome in an unselected population. J Clin Endocrinol Metab. 2004;89(6):2745-2749.

2. Mahmood M, El-Basel M, Sheta M. Polycystic ovary syndrome in premenopausal women with type 2 diabetes mellitus: prevalence, characters and related morbidity. Med J Cairo Univ. 2009;77(4):327-335.

3. Legro RS. Polycystic ovary syndrome and cardiovascular disease: a premature association? Endocr Rev. 2003;24(3):302-312.

4. Hardiman P, Pillay OS, Atiomo W. Polycystic ovary syndrome and endometrial carcinoma. Lancet. 2003;361(9371):1810-1812.

5. Ehrmann DA. Polycystic ovary syndrome. N Engl J Med. 2005;352(12): 1223-1236.

6. Fang YZ, Yang S, Wu G. Free radicals, antioxidants, and nutrition. Nutrition. 2002;18(10):872-879.

7. Essa MM, Braidy N, Waly MI, et al. Impaired antioxidant status and reduced energy metabolism in autistic children. Res Autism Spectr Disord. 2013;7(5):557-565.

8. Tapia-Vieyra JV, Delgado-Coello B, Mas-Oliva J. Atherosclerosis and cancer; a resemblance with far-reaching implications. Arch Med Res. 2017;48(1):12-26.

9. Tramutola A, Lanzillotta C, Perluigi M, Butterfield DA. Oxidative stress, protein modification and Alzheimer disease. Brain Res Bull. 2017;133:88-96.

10. Asmat U, Abad K, Ismail K. Diabetes mellitus and oxidative stress a concise review. Saudi Pharm J. 2016;24(5):547-553.

11. Costa S, Reina-Couto M, Albino-Teixeira A, Sousa T. Statins and oxidative stress in chronic heart failure. Rev Port Cardiol. 2016;35(1): 41-57.

12. Tošić-Pajić J, Šeklić D, Radenković J, et al. Augmented oxidative stress in infertile women with persistent chlamydial infection. Reprod Biol. 2017;17(2):120-125.

13. Agarwal A, Gupta S, Sharma RK. Role of oxidative stress in female reproduction. Reprod Biol Endocrinol. 2005;3:28.

14. Bansal AK, Bilaspuri GS. Impacts of oxidative stress and antioxidants on semen functions. Vet Med Int. 2011;2011:686137.

15. Lushchak VI. Free radicals, reactive oxygen species, oxidative stress and its classification. Chem Biol Interact. 2014;224:164-175.

16. Novo E, Parola M. Redox mechanisms in hepatic chronic wound healing and fibrogenesis. Fibrogenesis Tissue Repair. 2008;1(1):5.

17. Poston L, Igosheva N, Mistry HD, et al. Role of oxidative stress and antioxidant supplementation in pregnancy disorders. Am J Clin Nutr. 2011;94(Supp1_6):1980S-1985S

18. Hilali N, Vural M, Camuzcuoglu H, Camuzcuoglu A, Aksoy N. Increased prolidase activity and oxidative stress in PCOS. Clin Endocrinol (Oxf). 2013;79(1):105-110.

19. Papalou O, Victor VM, Diamanti-Kandarakis E. Oxidative stress in polycystic ovary syndrome. Curr Pharm Des. 2016;22(18):2709-2722.

20. Zhang R, Liu H, Bai H, et al. Oxidative stress status in Chinese women with different clinical phenotypes of polycystic ovary syndrome. Clin Endocrinol (Oxf). 2017;86(1):88-96.

21. Zuo T, Zhu M, Xu W. Roles of oxidative stress in polycystic ovary syndrome and cancers. Oxid Med Cell Longev. 2016;2016:8589318.

22. Murri M, Luque-Ramírez M, Insenser M, Ojeda-Ojeda M, EscobarMorreale HF. Circulating markers of oxidative stress and polycystic ovary syndrome (PCOS): a systematic review and meta-analysis. Hum Reprod Update. 2013;19(3):268-288.

23. Archibong AE, Rideout ML, Harris KJ, Ramesh A. Oxidative stress in reproductive toxicology. Curr Opin Toxicol. 2018;7:95-101.

24. Yeon Lee J, Baw C-K, Gupta S, Aziz N, Agarwal A. Role of oxidative stress in polycystic ovary syndrome. Curr Womens Health Rev. 2010; 6(2):96-107.
25. Lorenz LB, Wild RA. Polycystic ovarian syndrome: an evidence-based approach to evaluation and management of diabetes and cardiovascular risks for today's clinician. Clin Obstet Gynecol. 2007;50(1):226-243.

26. Al Khaduri M, Al Farsi Y, Al Najjar TAA, Gowri V. Hospital-based prevalence of polycystic ovarian syndrome among Omani women. Middle East Fertil Soc J. 2014;19(2):135-138.

27. Al-Fazari M, Sulaiman MA, Al-Farsi YM, Al-Khaduri MM, Waly MI. Assessment of risk factors related to nutrition and dietary intake among Omani women with polycystic ovarian syndrome. EC Nutrition. 2017; 12(1):18-28.

28. Sulaiman MA, Al-Farsi YM, Al-Khaduri MM, Waly MI, Saleh J, Al-Adawi S. Psychological burden among women with polycystic ovarian syndrome in Oman: a case-control study. Int $J$ Womens Health. 2017;9:897-904.

29. Sergeant E [homepage on the Internet]. Epitools Epidemiological Calculators 2016. AusVet Animal Health Services and Australian Biosecurity Cooperative Research Centre for Emerging Infectious Disease Online; 2015. Available from: http://epitools.ausvet.com.au. Accessed March 5, 2018.

30. Rotterdam ESHRE/ASRM-Sponsored PCOS Consensus Workshop Group. Revised 2003 consensus on diagnostic criteria and longterm health risks related to polycystic ovary syndrome. Fertil Steril. 2004;81(1):19-25.

31. World Health Organization. Waist Circumference and Waist-hip Ratio:Report of a WHO Expert Consultation, Geneva, 8-11 December 2008; 2011. Available from: http://apps.who.int/iris/bitstream/h andle/10665/44583/9789241501491_eng.pdf;sequence=1. Accessed February 18, 2018.

32. Mani H, Davies MJ, Bodicoat DH, et al. Clinical characteristics of polycystic ovary syndrome: investigating differences in White and South Asian women. Clin Endocrinol (Oxf). 2015;83(4):542-549.

33. Wijeyaratne $\mathrm{CN}$, Balen $\mathrm{AH}$, Barth JH, Belchetz PE. Clinical manifestations and insulin resistance (IR) in polycystic ovary syndrome (PCOS) among South Asians and Caucasians: is there a difference? Clin Endocrinol (Oxf). 2002;57(3):343-350.

34. Hahn S, Tan S, Elsenbruch S, et al. Clinical and biochemical characterization of women with polycystic ovary syndrome in North RhineWestphalia. Horm Metab Res. 2005;37(7):438-444.

35. Kim JJ, Choi YM, Cho YM, et al. Prevalence of elevated glycated hemoglobin in women with polycystic ovary syndrome. Hum Reprod. 2012;27(5):1439-1444.

36. Sharif E, Rahman S, Zia Y, Rizk NM. The frequency of polycystic ovary syndrome in young reproductive females in Qatar. Int $J$ Womens Health. 2017;9:1-10

37. Golbahar J, Al-Ayadhi M, Das NM, Gumaa K. Sensitive and specific markers for insulin resistance, hyperandrogenemia, and inappropriate gonadotrophin secretion in women with polycystic ovary syndrome: a case-control study from Bahrain. Int J Womens Health. 2012;4: 201-206.

38. Balen A. The pathophysiology of polycystic ovary syndrome: trying to understand PCOS and its endocrinology. Best Pract Res Clin Obstet Gynaecol. 2004;18(5):685-706.

39. Margis R, Dunand C, Teixeira FK, Margis-Pinheiro M. Glutathione peroxidase family - an evolutionary overview. FEBS J. 2008;275(15): 3959-3970.

40. Özer A, Bakacak M, Kuran H, et al. Increased oxidative stress is associated with insulin resistance and infertility in polycystic ovary syndrome. Ginekol Pol. 2016;87(11):733-738.

41. Lü JM, Lin PH, Yao Q, Chen C. Chemical and molecular mechanisms of antioxidants: experimental approaches and model systems. $J$ Cell Mol Med. 2010;14(4):840-860.

42. Couto N, Wood J, Barber J. The role of glutathione reductase and related enzymes on cellular redox homoeostasis network. Free Radic Biol Med. 2016;95(Suppl C):27-42

43. Wu G, Fang Y-Z, Yang S, Lupton JR, Turner ND. Glutathione metabolism and its implications for health. J Nutr. 2004;134(3):489-492. 
44. Forman HJ, Zhang H, Rinna A. Glutathione: overview of its protective roles, measurement, and biosynthesis. Mol Aspects Med. 2009; 30(1-2):1-12.

45. Lang CA, Mills BJ, Mastropaolo W, Liu MC. Blood glutathione decreases in chronic diseases. J Lab Clin Med. 2000;135(5):402-405.

46. Dinger Y, Akcay T, Erdem T, Ilker Saygili E, Gundogdu S. DNA damage, DNA susceptibility to oxidation and glutathione level in women with polycystic ovary syndrome. Scand J Clin Lab Invest. 2005;65(8): 721-728.

47. Manafikhi H, Drummen G, Palmery M, Peluso I. Total antioxidant capacity in beta-thalassemia: a systematic review and meta-analysis of case-control studies. Crit Rev Oncol Hematol. 2017;110:35-42.

48. Morera-Fumero AL, Díaz-Mesa E, Abreu-Gonzalez P, FernandezLopez L, Cejas-Mendez MD. Low levels of serum total antioxidant capacity and presence at admission and absence at discharge of a day/ night change as a marker of acute paranoid schizophrenia relapse. Psychiatry Res. 2017;249:200-205.

49. Mohamadin AM, Habib FA, Elahi TF. Serum paraoxonase 1 activity and oxidant/antioxidant status in Saudi women with polycystic ovary syndrome. Pathophysiology. 2010;17(3):189-196.

50. Massafra C, Gioia D, De Felice C, et al. Effects of estrogens and androgens on erythrocyte antioxidant superoxide dismutase, catalase and glutathione peroxidase activities during the menstrual cycle. $J$ Endocrinol. 2000;167(3):447-452.

51. Block-Abraham DM, Ke RW, Bloomer RJ. Impact of serum estradiol on biomarkers of oxidative stress in polycystic ovary syndrome and ovulatory women. Clin Med Insights Womens Health. 2012;5:CMWH. S9721.

52. Demirbag R, Yilmaz R, Erel O. The association of total antioxidant capacity with sex hormones. Scand Cardiovasc J. 2005;39(3):172-176.

53. Serviddio G, Loverro G, Vicino M, et al. Modulation of endometrial redox balance during the menstrual cycle: relation with sex hormones. J Clin Endocrinol Metabol. 2002;87(6):2843-2848.

54. Agarwal A, Aponte-Mellado A, Premkumar BJ, Shaman A, Gupta S. The effects of oxidative stress on female reproduction: a review. Reprod Biol Endocrinol. 2012;10(1):49.

55. Ogunro PS, Bolarinde AA, Owa OO, Salawu AA, Oshodi AA. Antioxidant status and reproductive hormones in women during reproductive, perimenopausal and postmenopausal phase of life. Afr J Med Med Sci. 2014;43(1):49-57.

56. Pejić S, Todorović A, Stojiljković V, et al. Antioxidant status and sex hormones in women with complex endometrial hyperplasia. Cell Mol Biol (Noisy-le-grand). 2016;62(11):51-56.
57. Michos C, Kiortsis DN, Evangelou A, Karkabounas S. Antioxidant protection during the menstrual cycle: the effects of estradiol on ascorbic-dehydroascorbic acid plasma levels and total antioxidant plasma status in eumenorrhoic women during the menstrual cycle. Acta Obstet Gynecol Scand. 2006;85(8):960-965.

58. Liao H, Zhou Q, Zhang Z, et al. NRF2 is overexpressed in ovarian epithelial carcinoma and is regulated by gonadotrophin and sex-steroid hormones. Oncol Rep. 2012;27(6):1918-1924.

59. Sastre-Serra J, Valle A, Company MM, Garau I, Oliver J, Roca P. Estrogen down-regulates uncoupling proteins and increases oxidative stress in breast cancer. Free Radic Biol Med. 2010;48(4):506-512.

60. Mobley JA, Brueggemeier RW. Estrogen receptor-mediated regulation of oxidative stress and DNA damage in breast cancer. Carcinogenesis. 2004;25(1):3-9.

61. Nguyen H, Syed V. Progesterone inhibits growth and induces apoptosis in cancer cells through modulation of reactive oxygen species. Gynecol Endocrinol. 2011;27(10):830-836.

62. Ahangarpour A, Heidari H, Mard SA, Hashemitabar M, Khodadadi A. Progesterone and cilostazol protect mice pancreatic islets from oxidative stress induced by hydrogen peroxide. Iran J Pharm Res. 2014;13(3): 937-944.

63. Al-Lawati JA, Jousilahti PJ. Prevalence and 10-year secular trend of obesity in Oman. Saudi Med J. 2004;25(3):346-351.

64. Al Riyami A, Elaty MA, Morsi M, Al Kharusi H, Al Shukaily W, Jaju S. Oman world health survey: part 1 - methodology, sociodemographic profile and epidemiology of non-communicable diseases in Oman. Oman Med J. 2012;27(5):425-443.

65. Alnohair S. Obesity in gulf countries. Int J Health Sci (Qassim). 2014;8(1):79-83.

66. James AM, Collins Y, Logan A, Murphy MP. Mitochondrial oxidative stress and the metabolic syndrome. Trends Endocrinol Metab. 2012;23(9):429-434.

67. Baskol G, Aygen E, Erdem F, et al. Assessment of paraoxonase 1, xanthine oxidase and glutathione peroxidase activities, nitric oxide and thiol levels in women with polycystic ovary syndrome. Acta Obstet Gynecol Scand. 2012;91(3):326-330.

68. Sabuncu T, Vural H, Harma M, Harma M. Oxidative stress in polycystic ovary syndrome and its contribution to the risk of cardiovascular disease. Clin Biochem. 2001;34(5):407-413.
International Journal of Women's Health

\section{Publish your work in this journal}

The International Journal of Women's Health is an international, peerreviewed open-access journal publishing original research, reports, editorials, reviews and commentaries on all aspects of women's healthcare including gynecology, obstetrics, and breast cancer. The manuscript management system is completely online and includes

\section{Dovepress}

a very quick and fair peer-review system, which is all easy to use. Visit http://www.dovepress.com/testimonials.php to read real quotes from published authors. 